LA-5629-MS

Informal Report

UC-32 and $34 \mathrm{c}$

Reporting Date: May 1974

Issued: June 1974

\title{
LINX: Library Interpretation of \\ Neutron X-Sections
}

by

Patrick D. Soran

DISTRIBUTION OF THIS DOCUMENT IS UNLIMITED. 
This report was prepared as an account of work sponsored by the Uniced States Government. Neither the United States nor the United States Atomic Energy Commission, nor any of their employees, nor any of their contractors, subcontrectors, or their employees, makes any warranty, express or implied, or assumes any legal liability or responsibility for the accuracy, completeness or usefulness of any information, apparatus, product or process disclosed, or represents that its use would not intringe privately owned rights.

This work supported by the U.S. Atomic Energy Commission, Division of Reactor Research and Development.

In the interest of prompt distribution, this LAMS report was not edited by the Technical Information staff.

Printed in the United States of America. Available from National Technical Information Service

U.S. Department of Commerce 5285 Port Royal Road

Springtield, Virginia 22151

Price: Printed Copy \$4.00 Microtiche \$1.45 
Patrick D. Soran

\section{ABSTRACT}

Th.e LINX code is used to merge oingle and multiisotope libraries into a single master library. The code is written in FORTRAN and produces a binary l1brary for both ISOTXS and BRKOXS files of the CCCC interface eystem.

\section{INTRODUCTION}

LINX is a computer code for merging neutron multigroup data libraries which have been written in the $\operatorname{cccc}^{1}$ interface file format. The present version of LiNX only processes version II of the IsoTXs ${ }^{1}$ interface file and both versions II and III of the BRKoxs ${ }^{1}$ interface file. The code was designed to operate on a CDC 7600 machine.

\section{DISCUSSION}

When calculating neutron multigroup cross sections from the ENDF/ $\mathrm{B}^{2}$ data system, one generally performs such operations a nuclide of materlal at a time. Because of the larger turn-around times assoclated with processing more than one nuclide, as well as a desire to scrutintze the output from such a multigroup calculation, processing the point-wise one nuclide at a time makes good sense. After one has completed a set of such calculations the various multigroup data sets reside on tapes which are not In a format suitable for direct CCCC interface utilization in such codes as TRIPLET ${ }^{3}$. To help the user create a single multigroup library for either the ISOTXS (Infinite dilution and $0^{\circ} \mathrm{K}$ multigroup data) or BRKOXS (self-slifelded or f-factor tables) data files, the LINX code has been developed.

The LINX code operates from one master disk or tape and merges the ISOTXS or BRKOXS data file onto the master tape. (There is a separate master library for the ISOTXS and BRKOXS data f1le,) LINX thus produces the correct file data and canipulation of various multigroup cross sections set with a minimum of user intervention. LINX was designed primarily to be used with the Los Alamos Scientific Laboratory code MINX. ${ }^{4}$

\section{GUIDE TO USER APPLICATION}

The present verison of LINX requires five input/output units, one of which is a card input unit (unit 5) and one which is a printer output unit (unit 6). The remaining three units concern the manipulation of the data:

$\begin{array}{ll}\text { Unit } 3 & \begin{array}{l}\text { 01d master unit to which data will } \\ \text { be added } \\ \text { Unit which contains data that is } \\ \text { to be added }\end{array} \\ \text { Unit } 8 & \begin{array}{l}\text { New master unit containing the } \\ \text { information of unit } 3 \text { with unit } \\ 4 \text { information added. }\end{array}\end{array}$

The user input requirements to the code are minimal. The only complicated input parameter to LINX is that which specifies the number of records to be skipped to reach the end of information of a particular isotope. At this foint it is assumed that the user of LINX is somewhat familar with CCCC interface files and in particular the LOtA vector. The skip parameter does have a defaulc value that is quite commonly used. 
Number of

Word on

Card

Name of

Variable

Comments

Control Integers (415)

1

NTYPE

1 - wish to merge Isotxs file

2

ICHM 0 - Do not merge fission

2 - wish to merge BRKOXS file spectrum data

1 - Do merge fission spectrum data

3

IEDIT

0 - Do not edit old master unit (unit 8 )

1 - Do edit old master unit (unit 8)

$4 \quad$ NRSP

Number of records to be skipped as described in reference 1 . The defaule value is equal to the number of isotopes times $[2$ + number of scattering types (e.g. elastic, $n, 2 n$, inelastic, and total)\}.

The entire program resides in core of a $\mathrm{CDC}$ 7600 and requires 5 to 15 seconds to execute. The code at LASL exists as an UPDATE file (LINX) with a back-up checksum tape XX010452 assigned to P. D. Soran, $\mathrm{T}-1$.

\section{REFERENCES}

1. B. M. Carmichae1, D. A. Menley, and D. R. Vondy, "Report of the Subcomittee on Standard Interface Files," prepared for the committee on Computer Code Cookdination and issued as Los Alamos Sclentific Laboratory report LA-S324-MS.

2. M. K. Drake, "Data Formats and Procejures for ENDF/B Neutron Cross -Sectioa Library," Brookhaven National Laboratory report BNL 50274 (T-601) (TID4500; ENOF-102, Vol.t (artober 1.970).

3. Wm. H. Reed, T. R. Hill, F. H. Brinkley and K. D. Lathrop, "TRIPLET: A Two-Dimensional, Multigroup, Trlangular Mesh, Planar Geometry, Explicit Transport Code," Los Alamos Scientific Laboratory report LA-5428-NS (October 1973).

4. C. R. Weisbin, P. D. Soran, R. J. LaBauve, D. R. Harris, and J. S. Hendricks, "MINX - A Mulitigroup Interpretation of Nuclear Cross Sections from ENDF/B," Los Alamos Scientiflc Laboratory report, to be published.

LASL IDENTIFICATION :

LP-0299

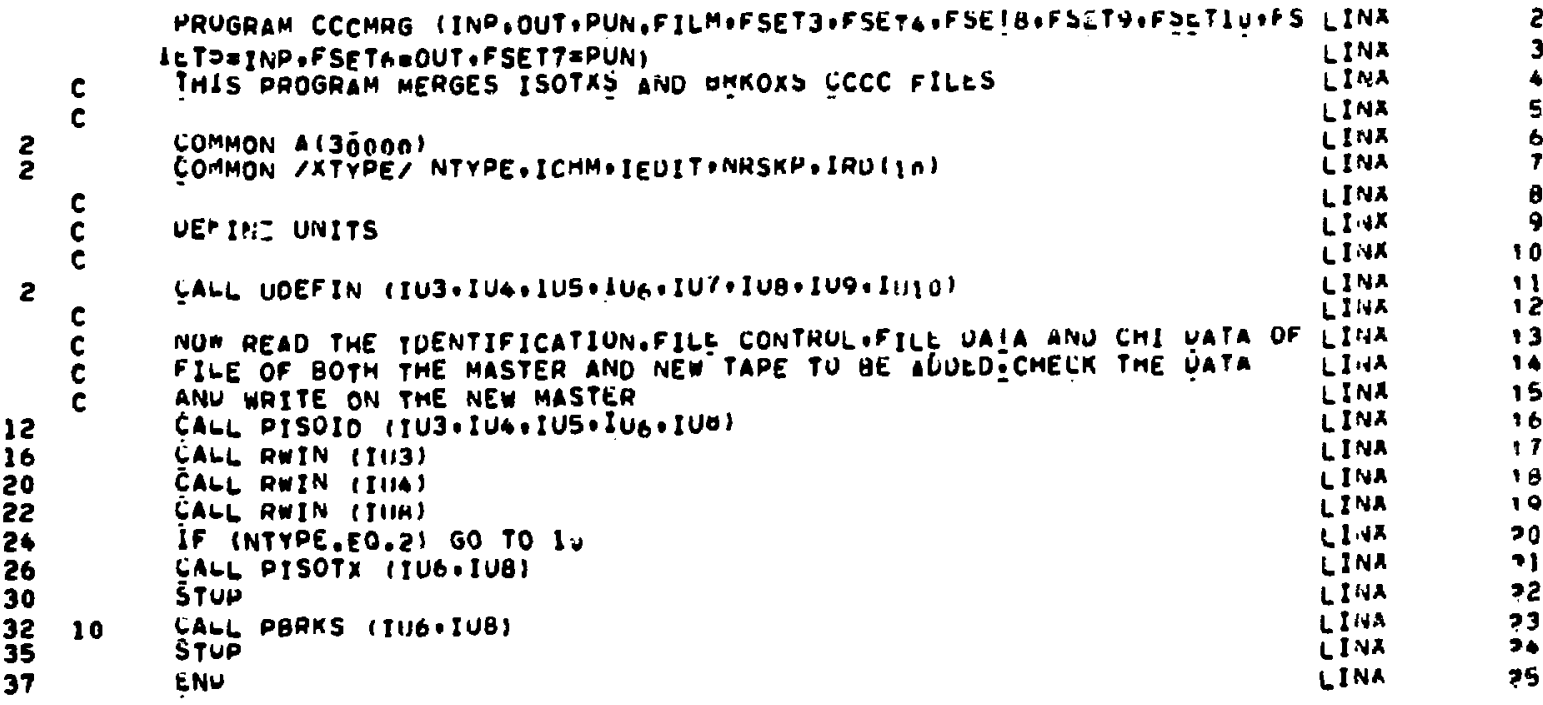




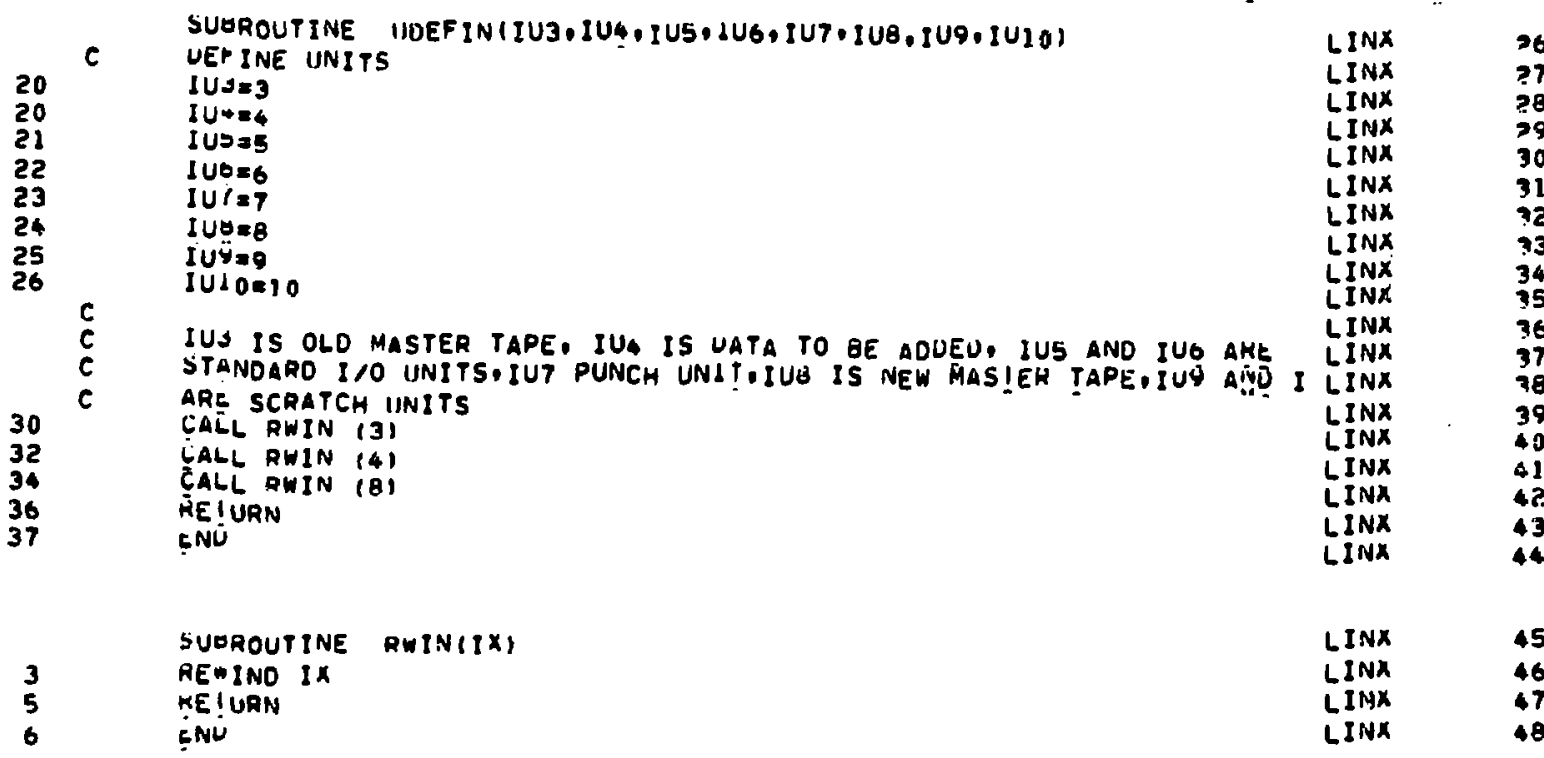

SUAROUTINE STYP\{IUS.IU6)

CUMMON /XTYPE, NFYPE,ICHMIIEOITONASKP,IRU (IT)

MEMD IIUS, IOINTYPE, ICHH, IED:T,NKSKP, (IHD (I), I I, IO)

WRITE IIUG, ZOINTYPE,ICHM,IEDIT,NHSKP, (IRU (I) I I. I0)

HE!UAN

SUOAOUTINE NISOIDIIU3,IUA,IUS,IUG,IUA)

UIAENSION 1750003 . O1250001. IM(1). I612)

LINX

$\operatorname{LINX}$

LivX

LINX

LINX

LINX

LINX

rommat (14T5)

LINX

FUMMAT IOI NTYPE-1/2=1SOTXS/GAKUXSE, IS.5X, OMERGE CHI UATA L/OAYS/ LINX

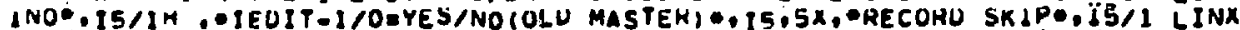

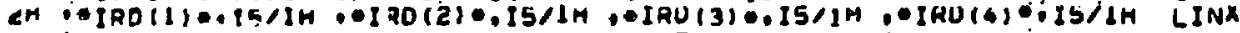

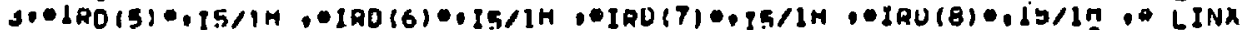
- IAU $(9) \bullet .15 / 1 N .0190(10) \cdot 34)$ ênu

\section{LINX}

LINX

UIMENSION CIOSOOOI, IC(1)

CUMMON $A, B, C$

COMHON IXTYPF, NTYPE ICNM, IEOIT INRSKP, IRUII İI

CUUIVALENCE $(A(2), I A(1)) \cdot(B(2), d t(1))$

EQUTVALECE (C, $(1)$.JCII)

C NEAO OPTIONS

CALL STYP (IUS.8U6)

ZRSCEI

[F (NTYRE.EO.Z) 60 TO \&U

C WOKK ON ISOTXS FILES

C MELO ANO COPY FILE TOENTIFICATIUN

CA-L AEEO (IIIA. IAEC,A(I),4,I)

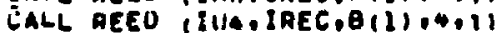

LALL aITE (IUR.jaEC AII) 4 II)

C REMU EILE CONTAOL FROM HASTER ANU NEW TAPE

IRECaIAEC+I

CALL GEEO II13.1AEC +A 11$) \cdot 9,1)$

iil $>0=\{a(2)$

NGMOLPPIA 111

MSLMAXIAIT)

IF (NRFKP.EQ.n) NRSKPENISU (2+NOCHAX)

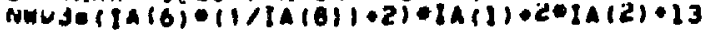

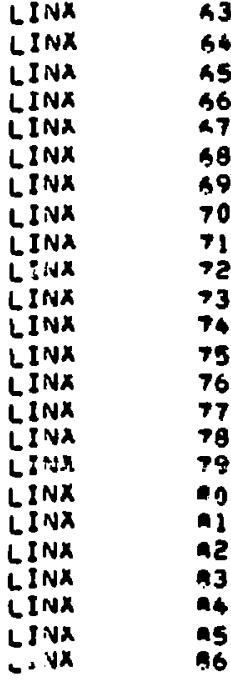




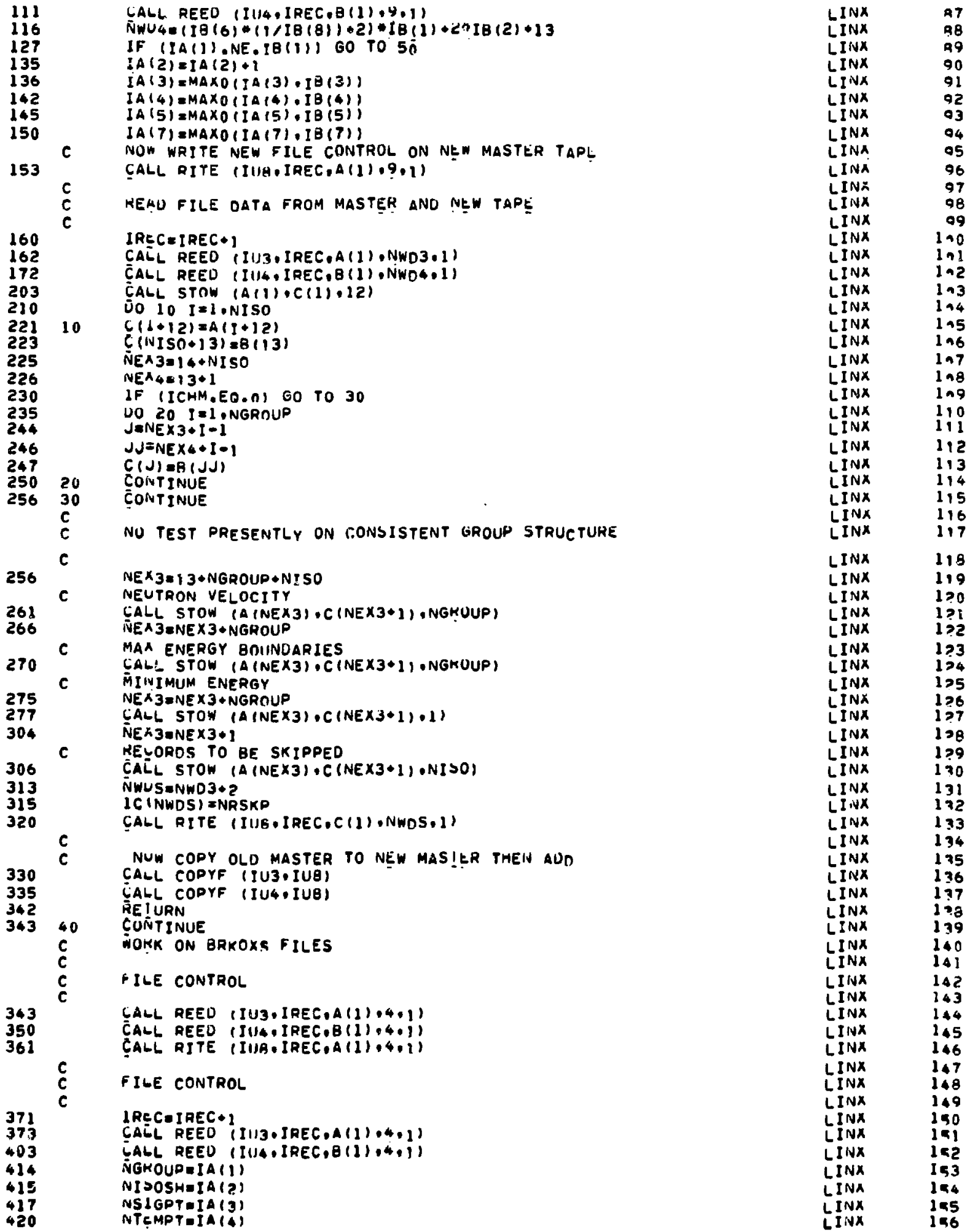




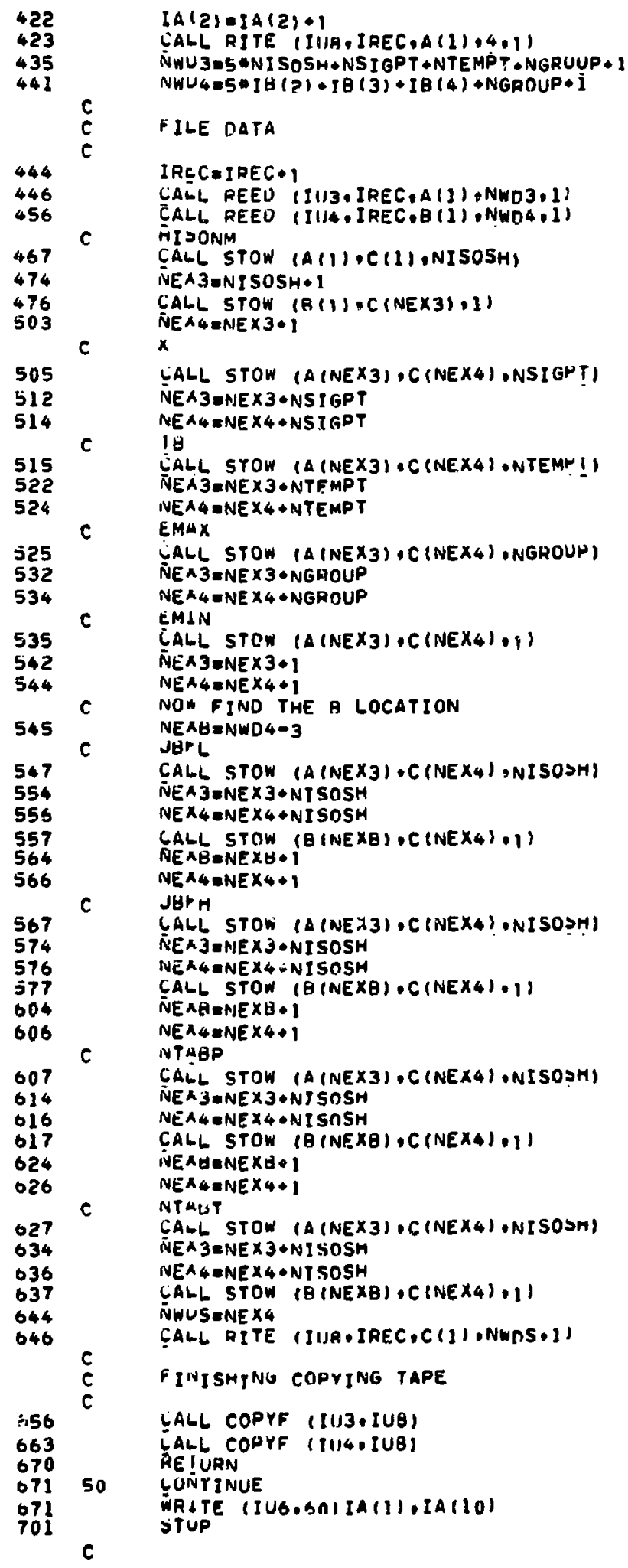

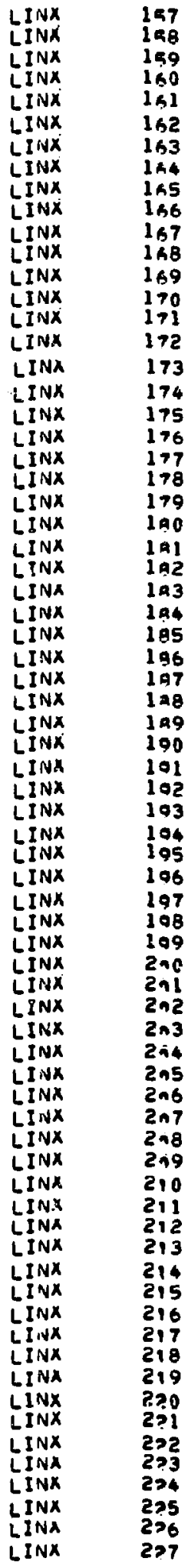


SUOROUTINE PISOTXINOUT,NA)

$\begin{array}{ll}6 & c \\ 6 \\ 6 \\ 6 \\ 6\end{array}$

C CONTROLS PRINTING OF THE ISOTXS PILE.

TMENSION MODE (3)

COMMON A (1)

UIMENSION IA (I)

COMMON /XTYPE, NTYPE,ICHM,IEDIY,NKSKP,IRD(1 in)

EQUIVALENCE (A) (1) IA I 1 )

UATA TOTAL,ELAS,AINEL . ANZN/GHTOTAL , GHELASTC,GHINELAS,GH NZN/

UAIA MODE (1) .MODE (2), MODE (3)/1,i,1)

DATA MO.MI,MQ10.1.98

CALL REED (N4.1.A(1).4,1)

$\bar{F} N=A(1)$

$M U=A(?)$

HS=A (3)

IVEREI I (4)

WRITE (NOUT, 450 ) FN, IVER,N4

WRITE (NOUT.460) HU.HS

c

45

55

56

60

61

63

64

66

67

71

76

106

117

121

126

132

140

146

151

152

167

170

174

200

213

220

224

250

252

257

303

305

315

317

324

352

354

361

363

--FILE CONTROL

IREC 1 = IREC $\mathbf{I}+1$

CALL REED (N4,IRECI, A (1), M9, MODE (1))

NGKOUPEIA (1)

NISOEIA (2)

MAXUP $=1 A(3)$

MAADN=IA (4)

MAAOROEIA(5)

ICIIIST $=I A(G)$

NSCMAX $=I A(7)$

NCËLOK=IA $(B)$

NSOLOKEIA $(\theta)$

IF (IRD (I).NE.IS GD TO 10

WRITE (NOUT, 240$)(I A(I), I=I, 9)$

C $=-F$ ILE DATA

io NWUSE (ICHIST* $(1 / N C S L O K)+2) * N G R O U P+2 * N I 5 O+13$

[REC I $=[R E C]+1$

CALL REED (NA, IRECI,A1I), NWOS, MUDE (I))

IF (IAO(2). NE.I) GO TO 40

WRITE (NOUY, 250$)(A(I), I=1,12$ )

WRITE (NOUT,260)

UU $20 I=1 \cdot N I S O$

$J=1+12$

20 WRITE (NOUT,270)I,A(J)

NEXTEI $13+$ NISO

IF (NCBLOK.GT.1) GO TO 30

WRITE (NOUT, DBO)

CALL WOT (AINEXT),ICHIST,NGROUP,MI.GHIN GP .6HOU! GP,MI,NOUT)

NEAT ONEXT + NGROUP I ICHIST

30 WRITE (NOUT -290)

CALL WOTE (AINEXT) ,NGROUP, A,MO,A,MO,A,MO,A,MO,A,MO,A,MO,A,MU,NUUT)

NEAT $=$ NEXT + NGROUP

WRLTE (NOUT B 3NO)

CALL WOTE (A (NEXT) ,NGROUP, A, MO, A,HO,A,MO,A,MO,A,MO, A, MO, A, MUU, NUUT)

NEAT WNEXT + NGROUP

WRLTE (NOUT.31O)A (NEXT)

NEXT $\triangle N E X T+1$

WR LTE (NOUT.320)

CSALL WOTB (A INEXT), NISO,A,MO,A,MO,A,MO,A,MR̈, A,MU,A,MO,A, MO,INOUI)

LINX

LINA

LINX

LINX

LINX

LINX

LINX

LINX

LINA

LINX

LINX

LINX

LINX

LINX

LINX

LINX

LINX

LINX

$\operatorname{LINX}$

LINX

LINX

LINX

LINX

LINX

LINX

LINX

IINX

LINA

LINX

LINX

LINX

LINX

$\operatorname{LINX}$

LINX

LINX

LINX

LINX

LINX

LINX

LINK

LINX

LINX

LINX

LINX

LINX

LINX

LINA

LINX

LINX

LINX

LINX

LINX

LINX

LINA

LINX

LINA

LINX

LINX

LINX

- -sEY CHI DATA

LINX

LINA

LINA

IF (NCBLOK.EO.1) GO TO OU

NWUSE ( (NGROUP-1) /NCELOK+1) ICHIST

LINA

LINA

NP! $=!$

UO $50 I=I$.NCAI. OK

LINX

LINX 


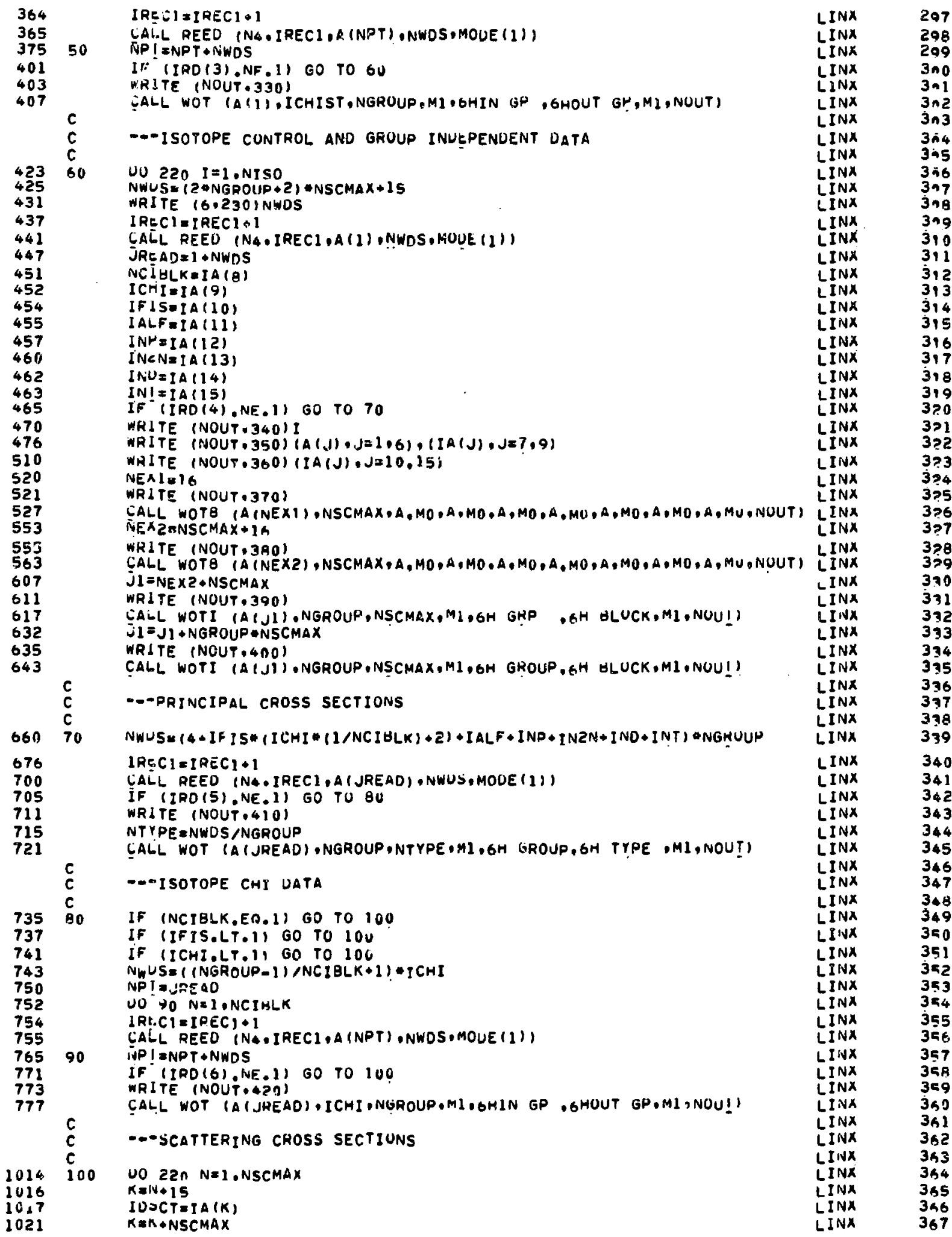




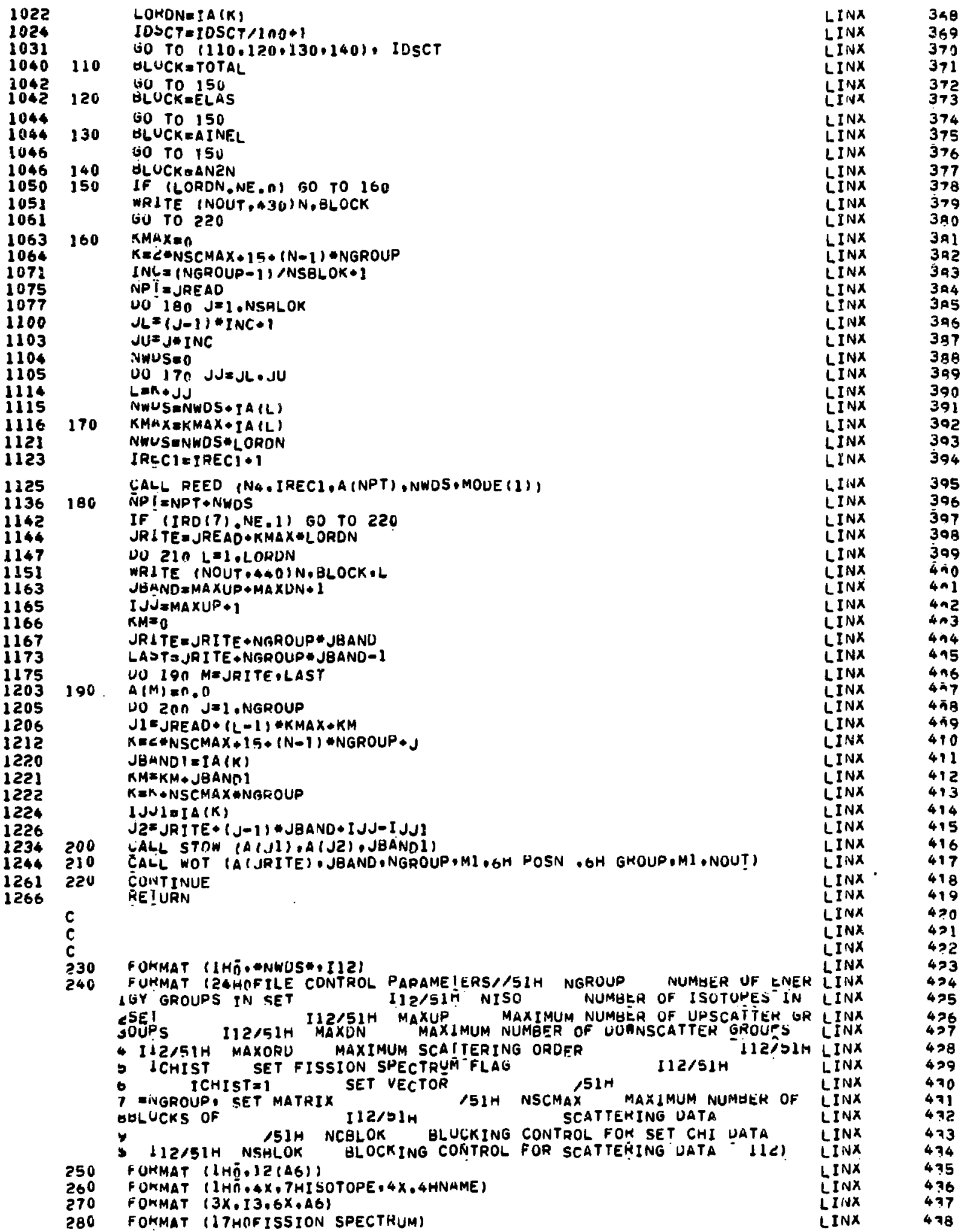


FOWMAT (33HOMEAN NEUTRON VELOCITY IN GROUP J/9X. IHJ)

$\operatorname{LINX}$

F UKMAT (2IHOMAXIMUM ENERGY BOUNÖ/3X,GHGROUP, IHJ) (INX

F UMMAT (28HOMTNIMUM ENERGŸ BOUNU OF SET//1GX,El2:5)

LINXX

F UMMAT (32HONIIMBER OF RECURDS TU HE SKIPPED//5X,7HISC (EEM)

FUKMAT (2IHOSET FISSION SPECTRUM)

346 FUWMAT (BHÖISOTOPE,I3)

FOKMAT 127HOISOTOPE CONTROL PARAMETERS/15IH IUPC LABEL

$7 X, A O / S I H$ AMASS GKAM ATOMIC WEIGHT LINX

SELLH/FISSION $E 12.5 / 51 H^{-5}$

$\operatorname{LINX}$ TOTAL THERMAL ENERGY Y I LINX (W. SEC/FISS)

LINX

4 /51H TEMP TEMPERATURE IDEG K)

$E \operatorname{LINX}$

$\$ 12.5 / 51 H$ SIGPOT AV POTENTIAL SCATTERING (BARNS/ATOM) OLH ADENS REFERENCE ATÓM DENS 1 ITY

$7 R$ ISOTOPE CLASSIFICATION

$\checkmark v$-UNDEF INED

$\checkmark$

?

$151 \mathrm{H}$

$6=$ COOLANT

$151 \mathrm{H}$

1511

I $12 / 51$ H

EI2.5/51H KB LINX

LINA

151H 2=FERTILE I=LLSSILE LINX $3=0$ THER ACTINIUE

LINX

/SIH LINX $4=F$ ISSION PROOUCT

$151 H$

LINX

TOK

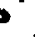

$112 / 51 \mathrm{H}$ $112 / 51 \mathrm{H}$ ICHI ISOTOPE FISSIOÑN SPECTRUM PLAG

151 NCIGLK ISOTUPE CHI HLOCKING FAC LINX

LINX ICHIDO USE SET CHI

LINX

$=1$ CHI VECTOR

FOKMAT 15IH

-NGROUP. CHI MATRIX

1 (N,F) X-SECTION PRESENT (IIEYES/UENO)

4PRESENT (IIYES/O=NO) IIZ/5IH INZN (N.ZN) X-SECTION PREESENT LINX

DIIIYES/OINO) IIZ/5IH IND (N.U) X-SECTION PRESENT IIIYYS/O LINX

OENU) $112 / 51 \mathrm{H}$ INT (N.T) X-SECTION PRESEN! (1YES/O=NO) LINX

7 II 2$)$

FUMMAT (28HOSCATTERING TYPE FOR HLOCK N/9X,IHN,6X,4HTYPE)

$\operatorname{LINX}$

FOHMAT (39HONUMBER OF SCATTERING ORUERS IN GLOCK N/QX, IHN,OX,4HTYP LINX bt)

F UKMA

IOTHOPOSITION OF IN-GROUP SCATTERING CROSS SECTION IN SCATT IERING DATA FOR GROUP U. SCATTERING BLOCK N

FUHMAT (2SHOPRINCIPAL CROSS-SECTIONS)

FONMAT (25HI)ISOTOPE FISSION SPECTRUMI

FOKMAT (GHÖBLOCK, I3,2X,A6ilgh SĈ̃TTERING OMITTEO)

FUHMAT (6HD̈BLOCK. I3.2X,A6,1BH SCATTERING, ORDER,13)

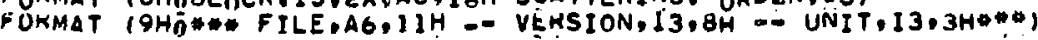

FOHMAT (24H *USER IDENTIFICATIUNN\#, AG:3X,46)

ENU

LINX

SUOROUTINE PHRKS (NOUT N N )

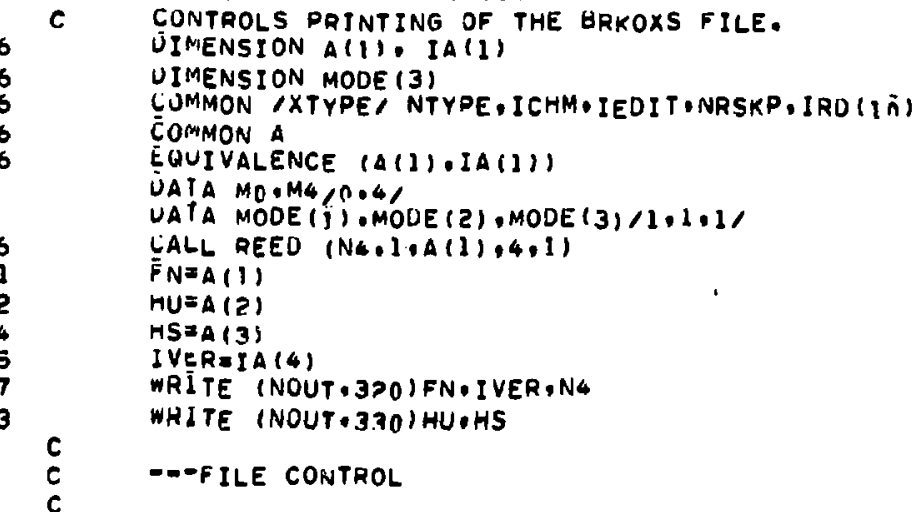

$\operatorname{LIN} x$

LINA

LINX

LINX

LINX

LINX

LINX

LINX

LINA

LINX

LINX

$\operatorname{LinX}$

LINX

LINX

LINX

LINA

LINX

LINX

LINA 


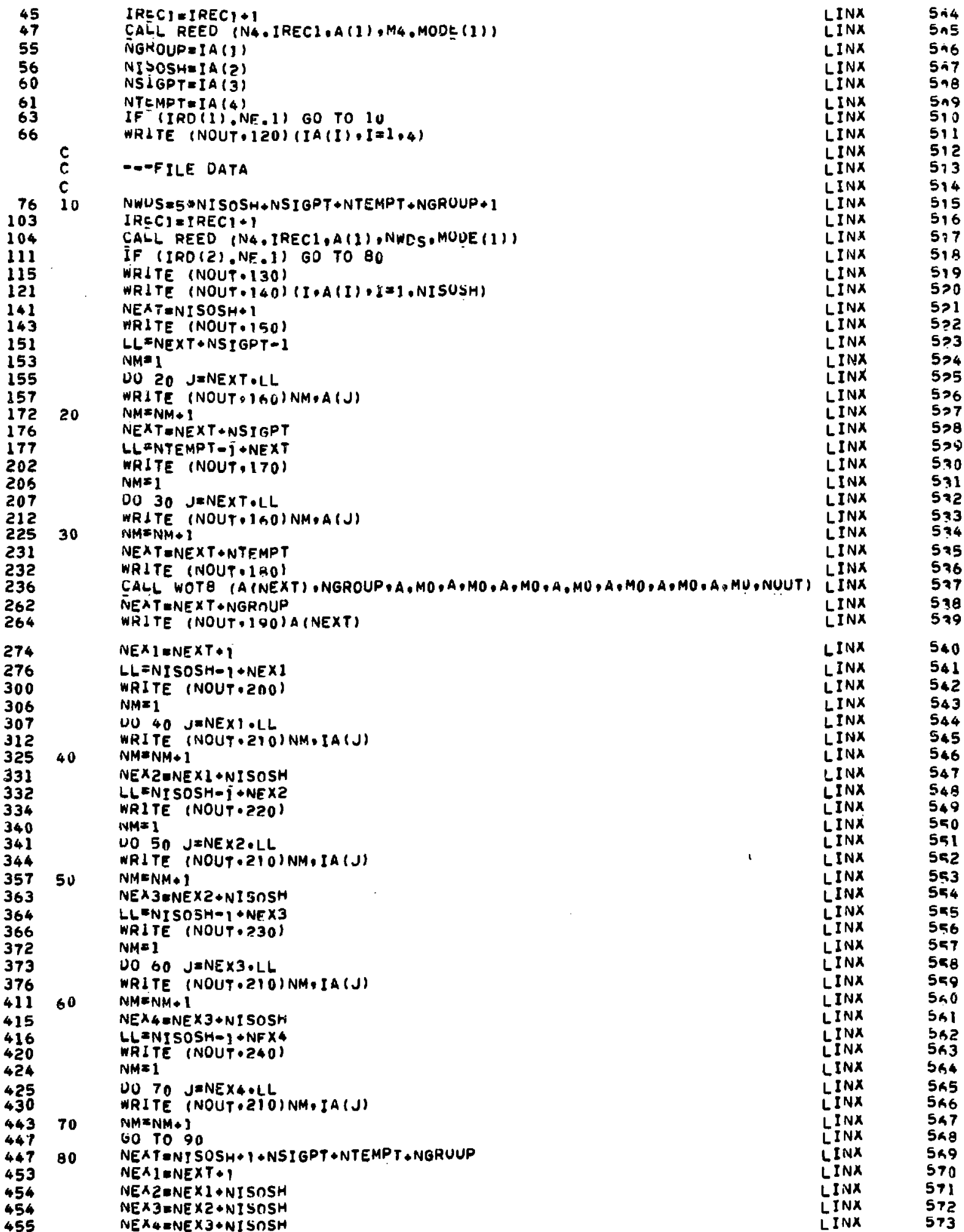


90 CUNTINUE

C $\quad-\infty$ SELF-SHIELDING FACTORS

LINX

LINX

LINX

JS PF $=N E \times 4+N I S O S H$

Vi $110 I=I$. NISOSH

LINX

NB INT $=1$ A (K)

$K \cong N E X_{4}-I * I$

$N B T E M=\lfloor A(K)$

$K=N E \times 1-1+I$

JURL:EIA(K)

$K=I V E \times 2-1+1$

$J \forall P H I=I A\{K\}$

NULFF $=J B F H I-J A F L I+1$

NPROENBINT *NBTEM $* N$ IFF

NWUS 5 \#NPRO

IF (NWDS.LE, n) 60 TO 100

IRECI IRECI I

CALL REEO (NA,IRECI,A (JSSF), NWUS, MODE (I))

IF (IRD (3), NE.1) GO TO 100

JEXJSSF+NPRO

$J 3=J 2+N P R O$

$J 4 \approx J 3 * N P R O$

$J 5=J G+N P R O$

WRITE (NOUT.250II

CALL WOT (A (JSSF) , NBINT, NUTEM,NUIFF, IHN, I HK, IHJ, NOUT)

WRLTE (NOUT,PSO)I

CALL WOT (A (JP) , NGINT , NBTEM,NDITF, INN, IHK,IHJ,NOUT)

WRITE (NOUTI?TO)I

CALL WOT (A $(J 3)$, NBINT, NETEM, NOIFF, IHN, LHK, I HJ NOUT)

WRITE (NOUT. PRO)I

CALL WOT (A J JW) , NEINT, NBTEM, NOIFF, I HN, IHK, IHJ,NOUT)

WRLTE (NOUT : ZYO) I

CALL WOT (AIJSI ONBINT ONBTEM,NOIFF, IHN, IHK, I HJ INOUT)

lou CONTINUE

667

C

067

671

673

700

704

705

706

707

710

712

716

731

771

--CROSS SECTIONS

NWUSEG*NGROUP

IRCCI $=[R E C I * 1$

CALL REED (NA,IRECI,A(JSSF), NWDS, MODE (2))

IF (IRD (4).NE. I) GO TO 110

$J Z=J 5 S F+N G R O U P$

$J 3=J Z+N G R O U P$

$J 4=J 3 \cdot N G R O U P$

JS JA $A$ NGROUP

J6E JS • NGROUP

WRITE (NOUT - 390)

WRITE (NOUT.310) (II I IE 1.6)

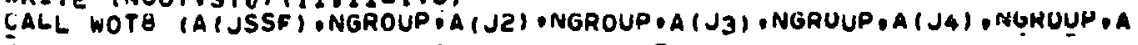

( (JD) .NGROUP,A (JG) , NGROUP, A,MO,A.MO,NOUT)

CONTINUE

REE!URN

c

FONMAT 124HREILE CONTROL PARAMETERS//SIH NGROUP NUMGEA UF ENEH LINA IGY GROUPS IN SET IIZ/SIM NISOSH HUMGEA OP ISOTUNES WIT LINA EM DELF- $\quad 151 \mathrm{H}$ SMIELLOING FACIORS 9 I12/5IH NSIGPT TOTAL NUMBER UF VALUES OF VAHIAGLE $X$ /5 LINR 4 IH WHICH ARE GIVEN. NSIGHT IS EJUAL TO /5IM $\checkmark$ THE SUM EROM I TO NISOSH OF NTAUP(I) IIZ/SIN NTEMPT TUTAL LINA

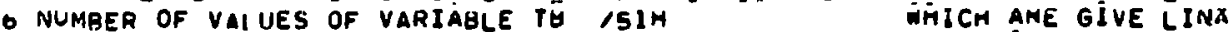
7N. NTEMPT IS EQUAL TO $/ 51 H$ THE SUM FRUM I TO NISUSA LINX GUF NTABTII) IIL)

FUKMAT (IHO,

LINX

LINX

FOMMAT $(5 \times, 13.7 \times, A 6)$

150

fokmat 141 HOL

I INX

$17 X$ ISHVALUE?

LINX

574

5ค!

SA2

$\sin 3$

5nc

5 as

5n 6

$5 n 7$

San

$5 a 9$

590

Sal

502

$\mathrm{SQ3}$

594

595

596

SQT

508

599

6a0

6nิ

$6 n 2$

$6 n 3$

6 ind

Gns

6ns

6n?

6aB

6 ng

610

611

612

613

614

615

616

617

618

619

620

621

$6 ? 2$

623

624

625

626

627

628

$6>9$

630

631

633

694

675

696

627

67.

679

640

641

642

643 


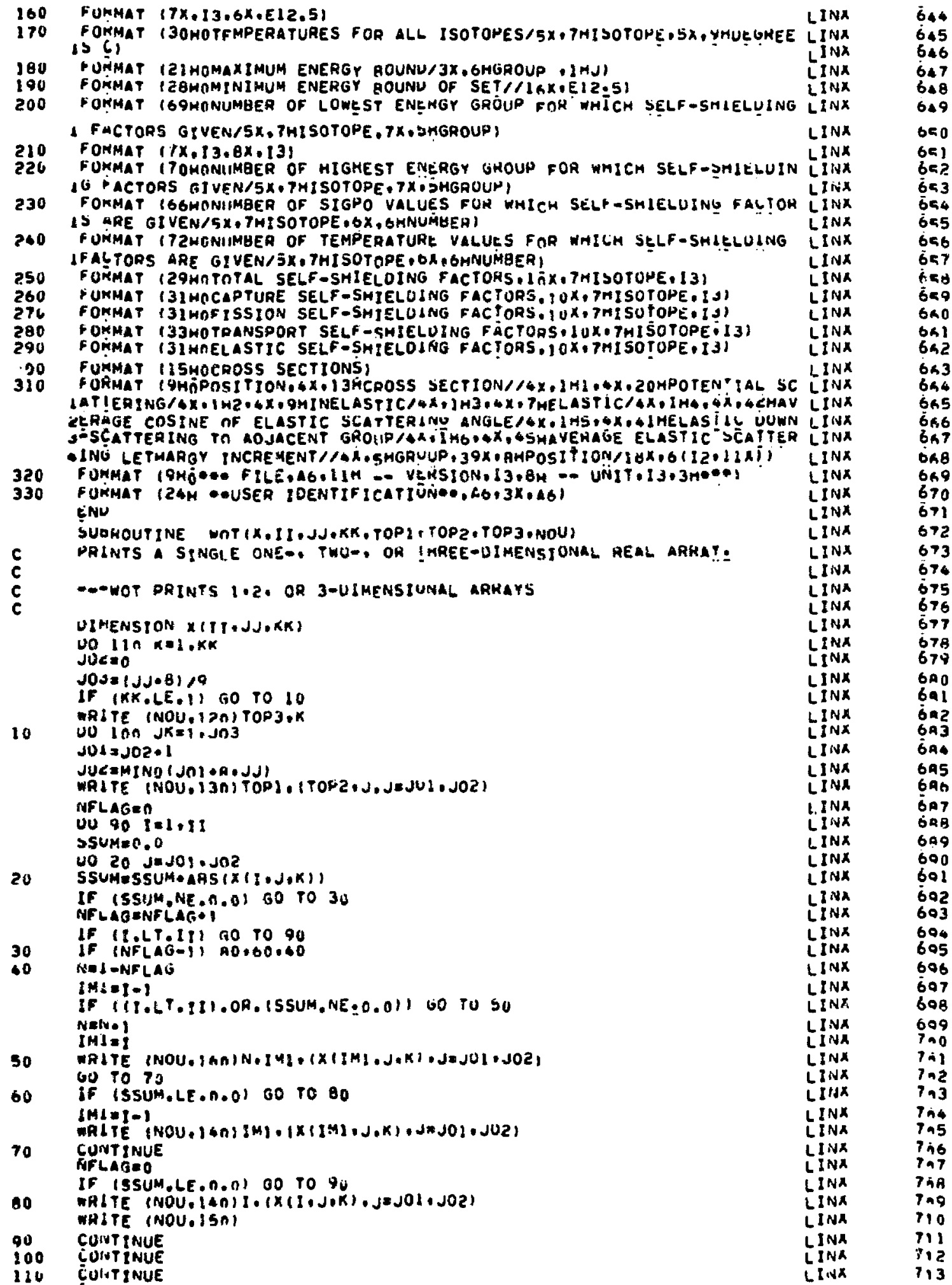




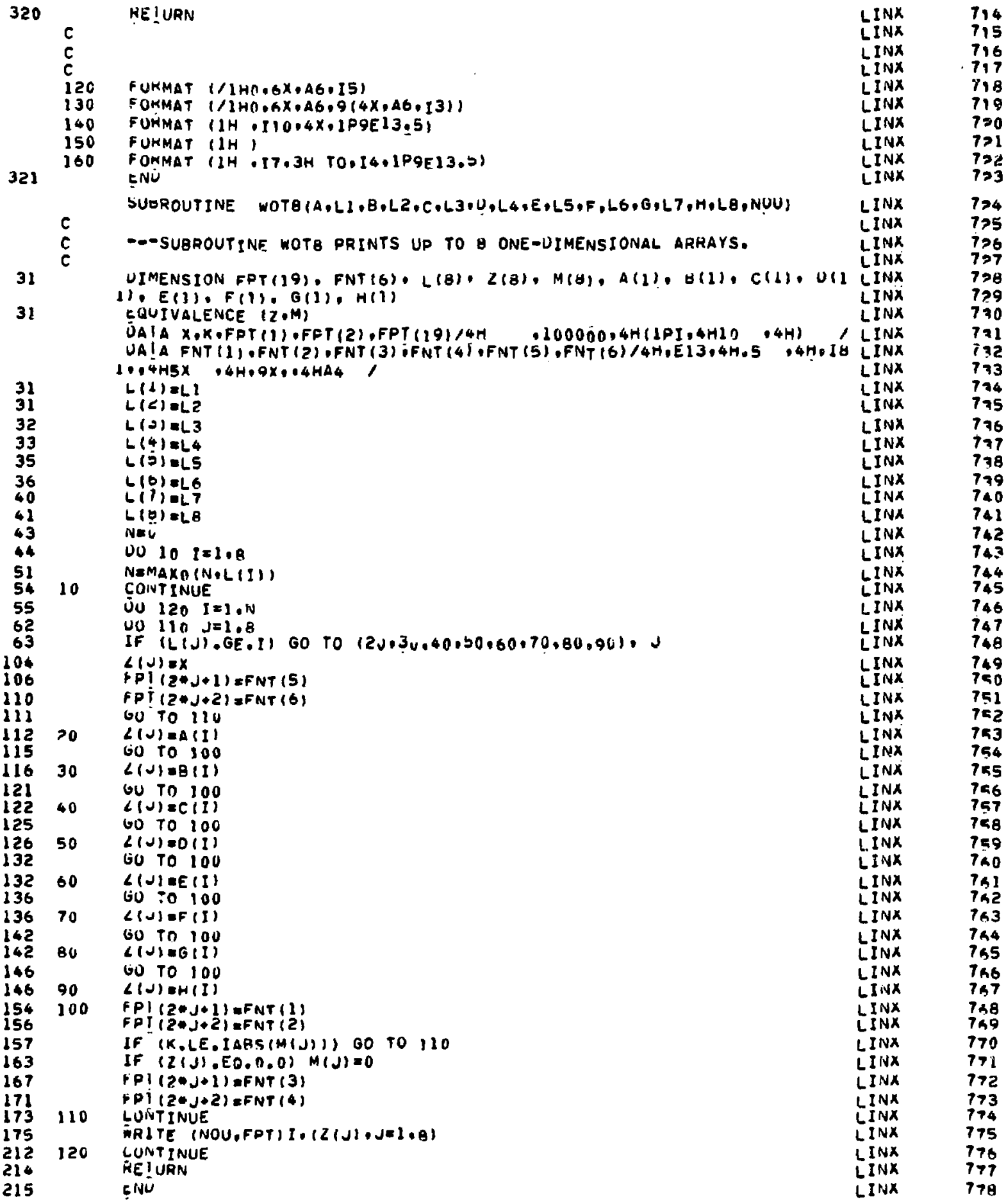


SUDHOUTINE WOTIIIX,II,JJOKK, TON I,TOPZ,TOP3,NOUI

$\operatorname{Lin} \pi$

LINX

$\operatorname{Lin} x$ UINENSION IXIII,JJPKKI UU $20 \mathrm{~K}=1 . K K$

$J U C=0$

Jos=(JJ+54)/?5

IF (KK.LE. I) RO TO 10

WRITE INOU.3NITOP3.K

10 UU $20 \mathrm{JK}=1 . \mathrm{J03}$

$\mathrm{JON}=\mathrm{J0S}+\mathrm{I}$

JUC $=M I N D(J n I+24 \cdot J \mathrm{~J})$

WRITE (NOU.4OITOPI -TOPZ, (J.JEJOL.JOZ)

No $20 \quad I=I \cdot I I$

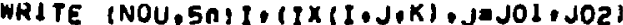

20 Cuivtinue

RE! UAN

c

30 FUKMAT $(1 / 2$ HO.6X,46.15)

40 FUKMAT $(/ 21 H N .16,1 H / .16 .2516)$

140

FOKMAT (1H , IH, TX,2514)

ENU

$\operatorname{lin}$

$\operatorname{Ling}$

LINA

Lind

INX

Link

LINX

LINX

Ling

LINA

LINA

LINA

LINA

LINA

LINX

LINA

LINA

LINA

Lind

SUOROUTINE HEED (N,IREC, X,L, MOOES)

LI INA

REIURN

ENU

Litidx

LinX

LINA

Liva

SUEROUTINE RITE(N,IREC, $X, L, M O D E)$

Lina

Lind

UIMENSION $\times$ (L

WRITE $(N)(X(I), I=1, L)$

HE! IURN

SNU

LINX

LINX

LINX

SUEROUTINE STOW (A,B,NWUS)

UIMENSION A (NWDS). B (NWOS)

IF (NWOS.LF. OI) RETURN

VU IO NEI.NWDS

$10 \quad \forall(N) \in A(N)$

RE IUAN

E़NU

$\operatorname{LINA}$

LINX

LINA

LINA

LINX

LINA

LINA

SUOROUTINE COPYPINA ONB.NGRQUP,NSCMAX,NISO,IRECI)

$\operatorname{Lin} \operatorname{Lin} x$

$8>6$

UIMENSION $\triangle(1), 1 A(1)$, MOUE (3)

$8>6$

COMMON A

EQUIVALENCE (a)(1).1A(1))

UAla MODE (1), MOUE $(2)$ - MOUE $(3), 1,1,1)$

LINA

$B>A$

insoloral

NO 60 I I I NISO

NWUSE (2*NGROUP +2$)$ NSCMAX +15

$I R E C I=I R E C I+I$

LALL REED (NAOIRECI,A(1) ONWDS, MUUE (1))

Lind

$4>9$

690

LinX

871

LINR

812

LINA

0.3

LINX

874 


\begin{tabular}{|c|c|c|c|c|}
\hline $\begin{array}{l}33 \\
51\end{array}$ & & 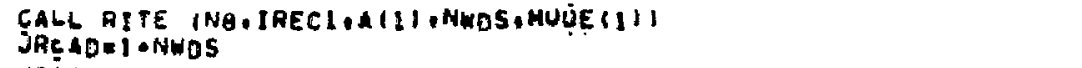 & $\begin{array}{l}\text { LINX } \\
\text { LINX }\end{array}$ & $\begin{array}{l}896 \\
897\end{array}$ \\
\hline $\begin{array}{l}52 \\
54 \\
55\end{array}$ & & 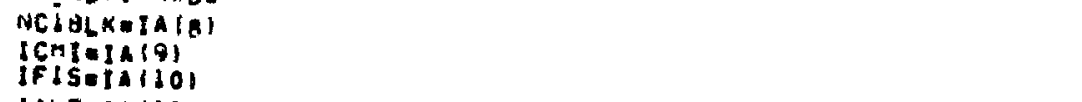 & $\begin{array}{l}\operatorname{LINX} \\
\operatorname{LINX} \\
\operatorname{LINX}\end{array}$ & $\begin{array}{l}898 \\
839 \\
840\end{array}$ \\
\hline $\begin{array}{l}57 \\
60\end{array}$ & & $\begin{array}{l}\text { IALFEIAIII) } \\
(N P=1 A T I S\}\end{array}$ & $\operatorname{lin} x$ & $\begin{array}{l}841 \\
842\end{array}$ \\
\hline $\begin{array}{l}62 \\
63 \\
65\end{array}$ & & 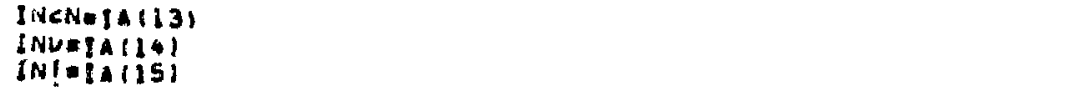 & $\begin{array}{l}\operatorname{LINX} \\
\operatorname{LINX} \\
\operatorname{LINX}\end{array}$ & $\begin{array}{l}843 \\
6 \$ 4 \\
845\end{array}$ \\
\hline & $\begin{array}{l}c \\
c\end{array}$ & - -PRINCIPAL CAOSS SECTIONS & $\begin{array}{l}\operatorname{LINX} \\
\operatorname{LINX} \\
\operatorname{LINX}\end{array}$ & $\begin{array}{l}846 \\
847 \\
849\end{array}$ \\
\hline $\begin{array}{r}66 \\
103\end{array}$ & & 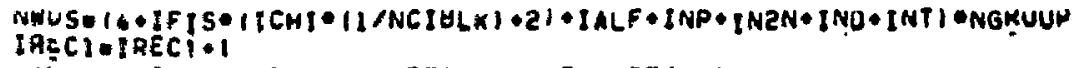 & $\operatorname{LinX}$ & $\begin{array}{l}849 \\
8090\end{array}$ \\
\hline 105 & $c$ & $\begin{array}{l}\text { LALL RITE ING,IRECIAAIJREADI ONWUS OMOOE IIUI } \\
\text {-O-ISOROPE CHI VAYA }\end{array}$ & $\begin{array}{l}\operatorname{LINX} \\
\operatorname{LINX} \\
\operatorname{LINX}\end{array}$ & $\begin{array}{l}8 \times 1 \\
8 \times 2 \\
8 \times 3\end{array}$ \\
\hline $\begin{array}{l}116 \\
122 \\
124 \\
126\end{array}$ & $C$ & 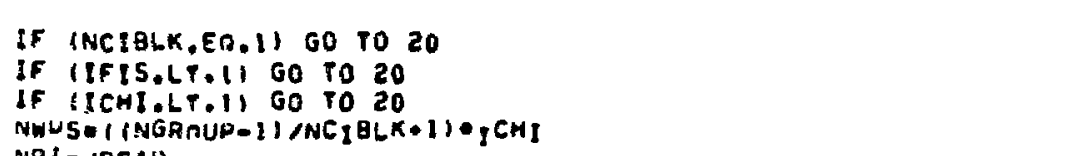 & $\begin{array}{l}\operatorname{LINX} \\
\operatorname{LINX} \\
\operatorname{LINX} \\
\operatorname{LINX} \\
\operatorname{LINX}\end{array}$ & $\begin{array}{l}8 \times 4 \\
8 \times 5 \\
8 \times 6 \\
8 \times 7 \\
8 \times 4\end{array}$ \\
\hline $\begin{array}{l}133 \\
135 \\
136 \\
137 \\
146 \\
165\end{array}$ & $c^{10}$ & 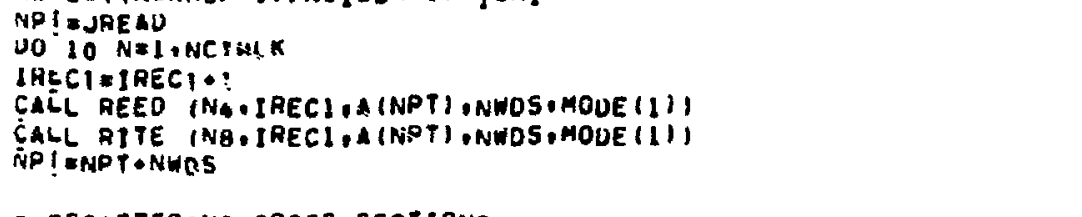 & $\begin{array}{l}\text { LINX } \\
\text { LINX } \\
\text { LINX } \\
\text { LINX } \\
\text { LINX } \\
\text { LINX } \\
\text { LINX }\end{array}$ & $\begin{array}{l}8<9 \\
8 \$ 0 \\
841 \\
8 \$ 2 \\
8,3 \\
464 \\
8 \$ 5\end{array}$ \\
\hline $\begin{array}{l}171 \\
173 \\
176\end{array}$ & $\begin{array}{l}c \\
c \\
c 0\end{array}$ & 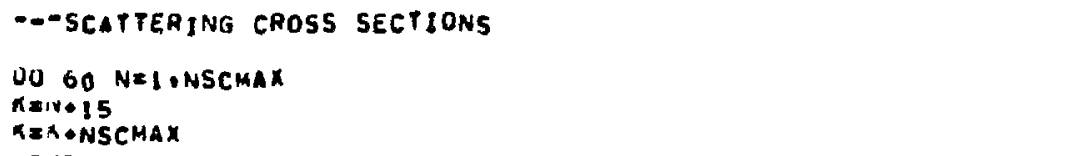 & $\begin{array}{l}\operatorname{LINR} \\
\operatorname{LINX} \\
\operatorname{LINX} \\
\operatorname{LINX} \\
\operatorname{LINX}\end{array}$ & $\begin{array}{l}\text { 8no } \\
\text { 8A7 } \\
\text { 8A8 } \\
\text { 8A9 } \\
\text { 870 }\end{array}$ \\
\hline $\begin{array}{l}175 \\
977 \\
201\end{array}$ & & $\begin{array}{l}\text { LOMDNEIAIK, } \\
\text { If } 1 \text { LORON, NE, th } 50 \text { TO } 30 \\
\text { SO TO } 60\end{array}$ & $\begin{array}{l}\operatorname{LINX} \\
\operatorname{LINX} \\
\operatorname{LINX}\end{array}$ & $\begin{array}{l}871 \\
872 \\
873\end{array}$ \\
\hline $\begin{array}{l}201 \\
202 \\
206 \\
212 \\
214\end{array}$ & 30 & 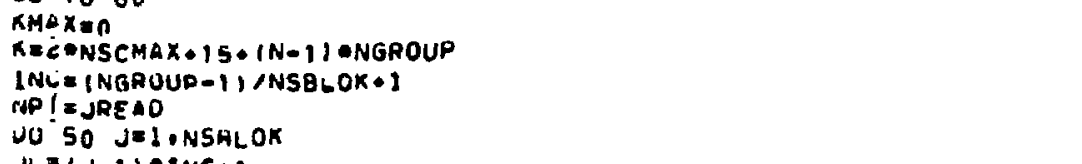 & $\begin{array}{l}\operatorname{Lin} x \\
\operatorname{LINA} \\
\operatorname{LINAX} \\
\operatorname{Lin} x \\
\operatorname{LIN} x\end{array}$ & $\begin{array}{l}874 \\
875 \\
876 \\
877 \\
878\end{array}$ \\
\hline $\begin{array}{l}215 \\
220\end{array}$ & & $\begin{array}{l}J U=j \in I N C \\
J=1 N-1): I N C \cdot 1\end{array}$ & $\operatorname{lin} x$ & $\begin{array}{l}679 \\
890\end{array}$ \\
\hline $\begin{array}{l}221 \\
222 \\
231\end{array}$ & & 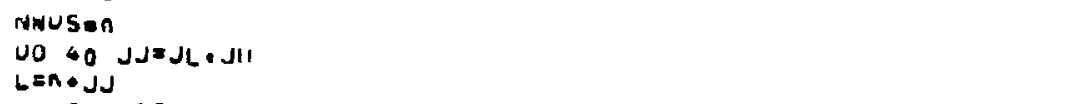 & $\begin{array}{l}\operatorname{Lind} \\
\operatorname{LINA} \\
\operatorname{LINA}\end{array}$ & $\begin{array}{l}891 \\
892 \\
8 \times 3\end{array}$ \\
\hline $\begin{array}{l}232 \\
233\end{array}$ & 40 & $\begin{array}{l}\text { NWUSENWOS }\{A I L\} \\
K M A X=K M A X+I A I L)\end{array}$ & $\operatorname{LINAx}$ & $\begin{array}{l}\text { B.94 } \\
\text { 8.95 }\end{array}$ \\
\hline $\begin{array}{l}242 \\
264 \\
245\end{array}$ & & 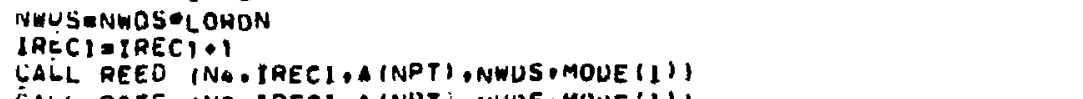 & $\begin{array}{l}\operatorname{lin} x \\
\operatorname{LINX} \\
\operatorname{LINX}\end{array}$ & $\begin{array}{l}896 \\
897 \\
\text { B98 }\end{array}$ \\
\hline 254 & 50 & $\begin{array}{l}\text { CALL RITE INA,IRECI,A INPTI N NHOS, MONE (I) } \\
\text { RPI INPT ONWOS }\end{array}$ & $\begin{array}{l}\text { LINR } \\
\text { LINA }\end{array}$ & $\begin{array}{l}8.99 \\
800\end{array}$ \\
\hline $\begin{array}{l}277 \\
304 \\
305\end{array}$ & 60 & $\begin{array}{l}\text { LUNTINUE } \\
\text { KE!UAN } \\
\text { CMU }\end{array}$ & $\begin{array}{l}\operatorname{LINX} \\
\operatorname{LINX} \\
\operatorname{LINX}\end{array}$ & $\begin{array}{l}801 \\
802 \\
803\end{array}$ \\
\hline $\begin{array}{l}6 \\
7\end{array}$ & & $\begin{array}{l}\text { SUDHOUTINE PIFIDIVOUT,NINS } \\
\text { RE!URN } \\
\text { CNU }\end{array}$ & $\begin{array}{l}\operatorname{LINX} \\
\operatorname{LINX} \\
\operatorname{LINX}\end{array}$ & $\begin{array}{l}894 \\
605 \\
496\end{array}$ \\
\hline
\end{tabular}

